This item was submitted to Loughborough's Research Repository by the author.

Items in Figshare are protected by copyright, with all rights reserved, unless otherwise indicated.

\title{
A viscous buoyancy-driven flow exhibiting finite-time blow-up
}

PLEASE CITE THE PUBLISHED VERSION

http://dx.doi.org/10.1093/imamat/hxs018

\section{PUBLISHER}

Oxford University Press on behalf of the Institute of Mathematics and its Applications / $\odot$ The Author

\section{VERSION}

AM (Accepted Manuscript)

\section{PUBLISHER STATEMENT}

This work is made available according to the conditions of the Creative Commons Attribution-NonCommercialNoDerivatives 4.0 International (CC BY-NC-ND 4.0) licence. Full details of this licence are available at: https://creativecommons.org/licenses/by-nc-nd/4.0/

\section{LICENCE}

CC BY-NC-ND 4.0

\section{REPOSITORY RECORD}

Kay, Anthony. 2019. "A Viscous Buoyancy-driven Flow Exhibiting Finite-time Blow-up”. figshare. https://hdl.handle.net/2134/16476. 


\title{
A viscous buoyancy-driven flow exhibiting finite-time blow-up
}

\author{
A. $\mathrm{KAY}^{*}$ \\ Department of Mathematical Sciences, Loughborough University, \\ Loughborough, Leicestershire, LE11 3TU
}

\begin{abstract}
We consider two-dimensional viscous flow driven by buoyancy forces resulting from a quadratic horizontal density variation in an unbounded domain between horizontal walls. The density is a quadratic function of the concentration of a tracer, so we solve a Navier-Stokes equation under the Boussinesq approximation, together with an advection-diffusion equation for the tracer. Stagnation-point similitude eliminates dependence on the horizontal coordinate. For the case of small Grashof number (large viscosity), the flow passes through three stages. A transient adjusts from the initial condition of static fluid to a "quasi-steady" regime in which buoyancy and viscous forces are in balance. The flow and temperature gradient slowly intensify until eventually the non-linear advection terms become dominant. The flow then enters its final phase, in which a more rapid intensification leads to a singularity in finite time. Analysis is by a combination of asymptotic methods and numerical computation. While no rigorous proof has been found that blow-up occurs, the numerical results support an asymptotic calculation premised on the occurrence of blow-up.
\end{abstract}

*Email: a.kay@lboro.ac.uk 


\section{Introduction}

This paper concerns the asymptotic structure of a two-dimensional, buoyancy-driven flow with stagnation-point similitude in a horizontally unbounded slab geometry. The flow is governed by the continuity equation

$$
\frac{\partial u_{*}}{\partial x_{*}}+\frac{\partial w_{*}}{\partial z_{*}}=0
$$

and the vorticity equation

$$
\frac{\partial \omega_{*}}{\partial t_{*}}+u_{*} \frac{\partial \omega_{*}}{\partial x_{*}}+w_{*} \frac{\partial \omega_{*}}{\partial z_{*}}=\frac{g}{\rho_{r}} \frac{\partial \rho_{*}}{\partial x_{*}}+\nu\left(\frac{\partial^{2} \omega_{*}}{\partial x_{*}^{2}}+\frac{\partial^{2} \omega_{*}}{\partial z_{*}^{2}}\right)
$$

in which the stars indicate dimensioned variables: $x_{*}$ and $z_{*}$ are horizontal and vertical coordinates, respectively, with $u_{*}$ and $w_{*}$ the corresponding velocity components; $\nu$ is the kinematic viscosity, $\rho_{*}$ is the density, and the vorticity is given by

$$
\omega_{*} \equiv \frac{\partial u_{*}}{\partial z_{*}}-\frac{\partial w_{*}}{\partial x_{*}}
$$

The Boussinesq approximation has been used in deriving (1.2). The density is assumed to depend only on the concentration $\theta_{*}$ of a conserved tracer, which obeys an advection-diffusion equation with diffusivity $\kappa$ :

$$
\frac{\partial \theta_{*}}{\partial t_{*}}+u_{*} \frac{\partial \theta_{*}}{\partial x_{*}}+w_{*} \frac{\partial \theta_{*}}{\partial z_{*}}=\kappa\left(\frac{\partial^{2} \theta_{*}}{\partial x_{*}^{2}}+\frac{\partial^{2} \theta_{*}}{\partial z_{*}^{2}}\right)
$$

A similarity solution of (1.2) is admitted when the horizontal density variation is quadratic. This is motivated by the approximately quadratic relation between temperature and density in fresh water near its temperature of maximum density (Oosthuizen \& Paul, 1996). Thus we assume the density to be a quadratic function of the tracer concentration $\theta_{*}$, referred to henceforth as "temperature":

$$
\rho_{*}=\rho_{r}-\alpha\left(\theta_{*}-\theta_{r}\right)^{2}
$$


where subscripts $r$ indicate constant reference values. A linear variation of temperature with the horizontal coordinate,

$$
\theta_{*}(x, z, t)=\theta_{r}+\lambda x_{*} \theta(z, t)
$$

then yields the quadratic horizontal density variation. We define further dimensionless (unstarred) variables, with the $x$-dependence removed by the stagnation-point similitude, as follows:-

$$
\begin{aligned}
z_{*} & =h z \\
t_{*} & =\left(\frac{2 g \alpha \lambda^{2} h}{\rho_{r}}\right)^{-1 / 2} t \\
u_{*}(x, z, t) & =\left(\frac{2 g \alpha \lambda^{2} h}{\rho_{r}}\right)^{1 / 2} x_{*} u(z, t) \\
w_{*}(x, z, t) & =\left(\frac{2 g \alpha \lambda^{2} h^{3}}{\rho_{r}}\right)^{1 / 2} w(z, t) \\
\omega_{*}(x, z, t) & =\left(\frac{2 g \alpha \lambda^{2}}{\rho_{r} h}\right)^{1 / 2} x_{*} \omega(z, t)
\end{aligned}
$$

in which $h$ is a vertical length scale. [Note that the factors of 2 were erroneously omitted from the corresponding equations in Grundy and Kay (2003).] The vorticity equation (1.2) and the temperature equation (1.4) then become

$$
\begin{aligned}
& \frac{\partial \omega}{\partial t}=-u \omega-w \frac{\partial \omega}{\partial z}-\theta^{2}+\operatorname{Gr}^{-1 / 2} \frac{\partial^{2} \omega}{\partial z^{2}} \\
& \frac{\partial \theta}{\partial t}=-u \theta-w \frac{\partial \theta}{\partial z}+\operatorname{Gr}^{-1 / 2} \operatorname{Pr}^{-1} \frac{\partial^{2} \theta}{\partial z^{2}} .
\end{aligned}
$$

Here the Grashof number, which characterises the relative strength of buoyancy and viscous forces, is defined by

$$
\mathrm{Gr}=\frac{2 g \alpha h^{5} \lambda^{2}}{\nu^{2} \rho_{r}},
$$

and the Prandtl number is the ratio of diffusivities of vorticity and heat,

$$
\operatorname{Pr}=\frac{\nu}{\kappa}
$$


The continuity equation and the definition of vorticity yield

$$
\omega=\frac{\partial u}{\partial z}=-\frac{\partial^{2} w}{\partial z^{2}}
$$

so that (1.12) and (1.13) can also be written in the forms

$$
\begin{aligned}
\frac{\partial^{3} w}{\partial t \partial z^{2}} & =\frac{\partial w}{\partial z} \frac{\partial^{2} w}{\partial z^{2}}-w \frac{\partial^{3} w}{\partial z^{3}}+\theta^{2}+\operatorname{Gr}^{-1 / 2} \frac{\partial^{4} w}{\partial z^{4}} \\
\frac{\partial \theta}{\partial t} & =\frac{\partial w}{\partial z} \theta-w \frac{\partial \theta}{\partial z}+\operatorname{Gr}^{-1 / 2} \operatorname{Pr}^{-1} \frac{\partial^{2} \theta}{\partial z^{2}}
\end{aligned}
$$

We shall assume rigid, no-slip, insulating boundaries at $z_{*}=0$ and $z_{*}=h$, so that in terms of the dimensionless variables we have

$$
w=0, \quad \frac{\partial w}{\partial z}=0 \quad \text { and } \quad \frac{\partial \theta}{\partial z}=0 \quad \text { at } \quad z=0 \text { and at } z=1
$$

The initial state is that the fluid is at rest with a uniform horizontal temperature gradient and no vertical temperature variation: in view of (1.6), these conditions may be written

$$
w=0 \text { and } \theta=1 \text { at } t=0 .
$$

The inviscid, non-diffusive version of this problem was studied by Jacqmin (1991) and Kay (1992). Jacqmin (1991) proved that a singularity is reached in finite time (the so-called blow-up phenomenon), and Grundy and Kay (2003) subsequently gave a detailed asymptotic analysis of the flow structure on the approach to this singularity. Jacqmin (1991) also considered a viscous, diffusive flow, but with the density as a conserved tracer which varied quadratically with the horizontal coordinate; his numerically computed results suggested that blow-up was occurring, but he was not able to support this with analytical calculations.

As with the abovementioned studies, our principal concern is to determine whether blowup occurs. Although we shall not provide a rigorous proof of blow-up, our asymptotic solutions for all stages of the flow yield strong evidence for blow-up. We shall concentrate on the limit of small Grashof number, i.e. very viscous flows: if blow-up occurs in this limit, 
we can be confident that it will occur for less viscous flows. There is also the incidental advantage that numerical solutions are easier in this limit: when the viscous/diffusive terms dominate the non-linear terms, standard methods for parabolic problems can be expected to work well. We briefly describe the numerical method in the next section of this paper, but our main focus is on asymptotic analysis. Thus we describe solutions for three regimes that apply at different stages of the flow in Sections 3 to 5, with the accuracy of the asymptotic solutions verified by comparison with the numerical results. We draw some conclusions and make suggestions for further work in Section 6.

\section{Numerical method}

With small Grashof number, standard numerical techniques for parabolic problems work well with (1.17) and (1.18): the nonlinear terms are relatively small, so the usual difficulties associated with nonlinear terms in numerical solutions of Navier-Stokes equations do not apply, except possibly close to blow-up. We use central-difference formulae for all spatial derivatives: in particular, third and fourth derivatives are approximated by

$$
\begin{aligned}
& \frac{\partial^{3} w}{\partial z^{3}} \approx \frac{1}{2 h^{3}}\left(w_{i+2}-2 w_{i+1}+2 w_{i-1}-w_{i-2}\right) \\
& \frac{\partial^{4} w}{\partial z^{4}} \approx \frac{1}{h^{4}}\left(w_{i+2}-4 w_{i+1}+6 w_{i}-4 w_{i-1}+w_{i-2}\right)
\end{aligned}
$$

where $h$ is the mesh size and subscripts indicate grid-point number.

Time-stepping is by Heun's method. However, in (1.17) this yields the value of $\partial^{2} w / \partial z^{2}$ at the next time step, so it is necessary to solve a tridiagonal system at each step to obtain the profile of $w$. For this purpose we may note that the $n \times n$ matrix $\left[a_{i j}\right]$ with elements

$$
a_{i j}=\left\{\begin{aligned}
-2 & \text { if } i=j \\
1 & \text { if } i=j \pm 1 \\
0 & \text { otherwise }
\end{aligned}\right.
$$


has the inverse $\left[b_{i j}\right]$ in which

$$
b_{i j}=-\frac{(n+1-i) j}{n+1} \quad \text { if } i \geq j ; \quad b_{j i}=b_{i j} .
$$

The results presented below were all obtained with $\mathrm{Gr}=10$ and $\operatorname{Pr}=1$; see the quasisteady solution in Section 3 below for an explanation of why 10 is effectively a "small" value of Grashof number. We used a grid of 200 uniformly spaced points across the domain, and then increased this to 400 and finally 800 when greater accuracy was needed on the final approach to blow-up; a Chebyshev series interpolation was used to obtain values of $w$ and $\theta$ at times when the grid was refined. The time step was $5 \times 10^{-7}$ for most of the integration, although a shorter time-step was employed during the initial transient and on the approach to blow-up. The usual checks on accuracy were performed, comparing results obtained with different values of grid spacing and time-step.

\section{Quasi-steady regime}

\subsection{Non-existence of a steady state}

If the flow does not reach a singularity in finite time, the alternatives are that it tends to a steady state or becomes unbounded as $t \rightarrow \infty$ (there being no reason to suspect that oscillatory or chaotic behaviour may occur). We now examine the possibility of a steady state. For this purpose it is useful to examine the quantity $\overline{\theta^{-1}}$, where the overbar indicates an integral over the space domain, i.e.

$$
\overline{f(\theta)} \equiv \int_{0}^{1} f(\theta) \mathrm{d} z
$$

Grundy and Kay (2003) observed that $\overline{\theta^{-1}}$ (which they denoted $I_{0}$ ) is invariant in inviscid flow, whereas from (1.18) and the boundary conditions (1.19) we obtain

$$
\frac{\mathrm{d} \overline{\theta^{-1}}}{\mathrm{~d} t}=-2 \mathrm{Gr}^{-1 / 2} \operatorname{Pr}^{-1} \int_{0}^{1} \theta^{-3}\left(\frac{\partial \theta}{\partial z}\right)^{2} \mathrm{~d} z .
$$


It can easily be verified that $\theta$ must remain strictly positive everywhere, so $\overline{\theta^{-1}}$ is monotonic decreasing. Furthermore, a steady state can only be reached if $\theta$ becomes uniform in $z$. But uniformity of $\theta$ reduces (1.13) to

$$
\frac{\partial \theta}{\partial t}=-u \theta
$$

so that $\theta$ can only remain uniform if $u$ is uniform. This in turn means $\omega \equiv 0$, but (1.12) shows that this state cannot persist as long as $\theta \not \equiv 0$. Hence the steady state is unachievable for any non-trivial initial condition on $\theta$.

\subsection{Quasi-steady solution at times $t=O(1)$}

Having shown that no steady-state solution exists, the obvious next step is to seek a steadystate solution. For small Grashof number, the leading-order balance in the vorticity equation (1.17) is between the buoyancy and viscous terms:

$$
\mathrm{Gr}^{-1 / 2} \frac{\partial^{4} w}{\partial z^{4}} \approx-\theta^{2}
$$

so that $w=O\left(\mathrm{Gr}^{1 / 2}\right)$. In order for the diffusive term in the temperature equation (1.18) to be balanced by the first nonlinear term, we then require that $z$-derivatives of $\theta$ be smaller than the absolute value of $\theta$ by a factor of order Gr. In view of the initial condition (1.20), we therefore write

$$
\theta=1+\operatorname{Gr} \theta_{1}(z)
$$

so that the leading-order solution of (3.4) with the boundary conditions (1.19) is

$$
w \approx-\frac{1}{24} \operatorname{Gr}^{1 / 2} z^{2}(1-z)^{2}
$$

while the leading-order balance in (1.18) is

$$
\operatorname{Gr}^{1 / 2} \operatorname{Pr}^{-1} \frac{\partial^{2} \theta_{1}}{\partial z^{2}} \approx-\frac{\partial w}{\partial z}
$$


Integrating (3.7) with $w$ given by (3.6) and applying the insulating boundary conditions from (1.19) leaves an undetermined constant in the solution for $\theta_{1}$. However, (1.18) and the boundary conditions yield

$$
\frac{\mathrm{d} \bar{\theta}}{\mathrm{d} t}=-2 \int_{0}^{1} w \frac{\partial \theta}{\partial z} \mathrm{~d} z=O\left(\mathrm{Gr}^{3 / 2}\right)
$$

so that

$$
\frac{\mathrm{d} \overline{\theta_{1}}}{\mathrm{~d} t}=O\left(\mathrm{Gr}^{1 / 2}\right)
$$

i.e. $\overline{\theta_{1}}$ varies slowly. Hence we may impose the condition $\overline{\theta_{1}}=0$ while $t=O(1) ;(3.7)$ then has the steady solution

$$
\theta_{1} \approx \theta_{1 s}=\frac{\operatorname{Pr}}{1440}\left(12 z^{5}-30 z^{4}+20 z^{3}-1\right)
$$

\subsection{Quasi-steady solution at large times}

The slow growth indicated by (3.9) shows that the solutions in Section 3.2 will not remain

valid at times of order $\mathrm{Gr}^{-1 / 2}$. However, for very viscous flow the spatial variations in $\theta$ across the domain are expected to remain small compared to its mean value, so we generalise (3.5) to

$$
\theta(z, t)=\bar{\theta}(t)+\operatorname{Gr} \theta_{1}(z, t)
$$

Hence (3.6) is replaced by

$$
w \approx-\frac{1}{24} \operatorname{Gr}^{1 / 2} \bar{\theta}^{2} z^{2}(1-z)^{2}
$$

as the solution of (3.4). This solution is quasi-steady in the sense that, although $\bar{\theta}$ varies in time, the solution was obtained while ignoring the time-derivative in (1.17): because of the large viscosity, the velocity profile adjusts effectively instantaneously to the variations in the buoyancy forcing. Neglect of the time-derivative can be justified a posteriori by noting from (3.12) and (3.8) that

$$
\frac{\partial^{3} w}{\partial t \partial z^{2}}=O\left(\mathrm{Gr}^{2}\right)
$$


Using (3.11) in (1.18) gives at leading order

$$
\operatorname{Gr}^{1 / 2} \operatorname{Pr}^{-1} \frac{\partial^{2} \theta_{1}}{\partial z^{2}} \approx-\bar{\theta} \frac{\partial w}{\partial z}
$$

in place of (3.7). With $\theta_{1}$ defined by (3.11) rather than (3.5) the condition $\overline{\theta_{1}}=0$ is exact, so (3.14) has the quasi-steady solution

$$
\theta_{1} \approx \theta_{1 q}=\frac{\operatorname{Pr}}{1440} \bar{\theta}^{3}\left(12 z^{5}-30 z^{4}+20 z^{3}-1\right) .
$$

Substitution into (3.8) then yields

$$
\frac{\mathrm{d} \bar{\theta}}{\mathrm{d} t} \approx \frac{\mathrm{Gr}^{3 / 2} \operatorname{Pr}}{181440} \bar{\theta}^{5}
$$

and with the initial condition $\bar{\theta}=1$ at $t=0$ we obtain

$$
\bar{\theta} \approx\left(1-\frac{\mathrm{Gr}^{3 / 2} \mathrm{Pr}}{45360} t\right)^{-1 / 4}
$$

The large numerical factor dividing $\mathrm{Gr}^{3 / 2}$ justifies taking 10 as a "small" value of Grashof number in the numerical calculations.

We may complete our quasi-steady analysis by substituting (3.17) into the solutions (3.12) for $w$ and (3.15) for $\theta_{1}$. The solution (3.17) shows slow but accelerating growth, with eventual blow-up at time $t=45360 \mathrm{Gr}^{-3 / 2} \mathrm{Pr}^{-1}$. This estimate of blow-up time is expected to be accurate to leading order, although the above solution is expected to fail close to blowup, when non-linear advection terms and possibly time derivatives in (1.17) and (1.18) will become large.

\section{The initial transient}

Our quasi-steady solutions for $w$ and $\theta$ do not satisfy the initial conditions (1.20), so there must be a transient by which the flow adjusts from its initial state to the buoyancy-viscous balance. So we solve

$$
\frac{\partial^{3} w}{\partial t \partial z^{2}}=1+\operatorname{Gr}^{-1 / 2} \frac{\partial^{4} w}{\partial z^{4}}
$$


noting that the transient will be fast (on a time-scale of order $\mathrm{Gr}^{1 / 2}$ ) so that we can write $\theta \approx 1$ in (1.17), cf. Section 3.2 above. We now obtain

$$
w \approx \operatorname{Gr}^{1 / 2}\left\{-\frac{1}{24} z^{2}(1-z)^{2}+\sum_{p=0}^{\infty} \frac{1-\cos ((4 p+2) \pi z)}{8(2 p+1)^{4} \pi^{4}} \exp \left(-(4 p+2)^{2} \pi^{2} \mathrm{Gr}^{-1 / 2} t\right)\right\}
$$

valid at all times $t \leq O(1)$. The development of the transient in $w$, with comparisons between numerically computed results, the transient solution (4.2) and the steady solution (3.6), is shown in Figure 1. Results computed from the full equations (1.17) and (1.18) show a small but just noticeable difference from the transient solution at time $t=0.05$, whereas the difference is no longer visible at $t=0.1$. At $t=1$, when the slowest-decaying $(p=0)$ mode in the transient in (4.2) has decay exponent $-4 \pi^{2} \mathrm{Gr}^{-1 / 2} t \approx-12.48$, the numerical solution (not shown in Figure 1) is indistinguishable from the steady solution (3.6). This indicates that the subsequent slow variation of $\bar{\theta}$ from its initial value of unity has not had any appreciable effect on $w$ at this time.

Turning to the temperature equation (1.18), the leading-order balance while $t=O\left(\mathrm{Gr}^{1 / 2}\right)$ is

$$
\operatorname{Gr} \frac{\partial \theta_{1}}{\partial t}-\operatorname{Gr}^{1 / 2} \operatorname{Pr}^{-1} \frac{\partial^{2} \theta_{1}}{\partial z^{2}}=\frac{\partial w}{\partial z}
$$

noting that (3.5) applies at this time. The term on the right-hand side of (4.3) operates as a forcing, and is found from (4.2). Thus the transient part of the solution for $\theta_{1}$ includes responses to both the steady and transient parts of $\partial w / \partial z$ : we may write

$$
\theta_{1}=\theta_{1 s}+\theta_{t s}+\theta_{t t}
$$

in which $\theta_{1 s}$ is the steady solution (3.10), while the transient terms $\theta_{t s}$ and $\theta_{t t}$ are rather cumbersome and so are given in the Appendix. Comparing the numerically computed profile of $\theta$ at time $t=0.05$ with that obtained from our analytical solution at $O(\mathrm{Gr})$ (i.e. (3.5) with $\theta_{1}$ given by (4.4) with (3.10), (A.1) and (A.2)), we see from Figure 2(a) that there is a considerably greater discrepancy than there is between the $w$ profiles at the same time in 


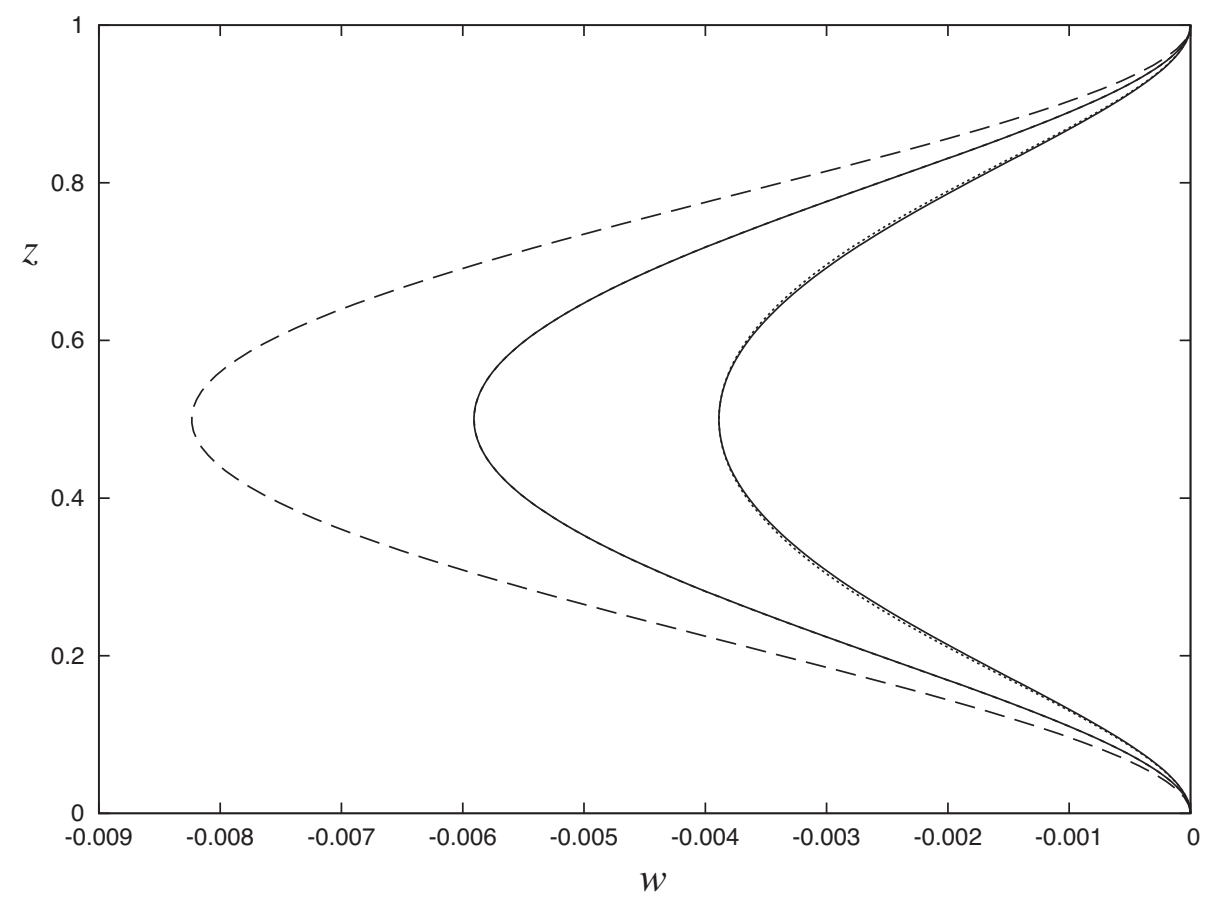

Figure 1: Profiles of $w$ at times $t=0.05$ and $t=0.1$ during the transient. Solid curves show results of numerical computation; dotted curves are plotted from formula (4.2) (although the dotted and solid curves are indistinguishable at $t=0.1$ ). Also shown as a dashed curve is the quasi-steady formula (3.6) 
(a)

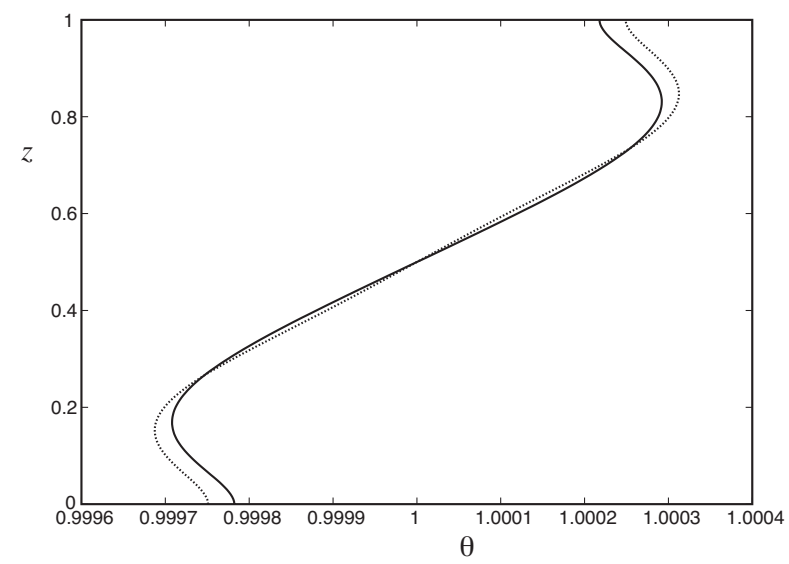

(b)

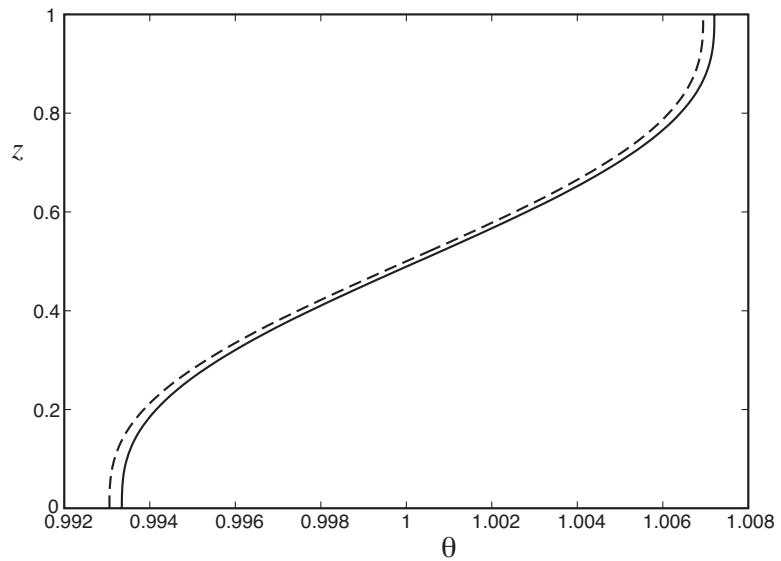

Figure 2: Profiles of $\theta$ at times (a) $t=0.05$ and (b) $t=2$. Solid lines: numerical solution of full equations (1.17) and (1.18). Dotted line: transient at $t=0.05$, calculated by solving (4.3). Dashed line: steady solution (3.10).

Figure 1. Furthermore, at time $t=2$ when the transient has died away (the slowest decaying mode is $m=0$ in (A.1) and (A.2), with decay exponent $-\pi^{2} \operatorname{Pr}^{-1} \mathrm{Gr}^{-1 / 2} t \approx-6.242$ at $t=2$ ), there remains a significant discrepancy, now uniform over the domain, between the numerical solution and the steady solution (3.10): see Figure 2(b).

Now, $\theta_{1}$ only accounts for the spatial variation of $\theta$ about its mean (note that equation (4.3) and the boundary conditions imply that $\mathrm{d} \overline{\theta_{1}} / \mathrm{d} t=0$ at all times), whereas the spatially uniform discrepancy implies that we have failed to capture an additive term. It is tempting to simply replace (3.5) with (3.11), with $\bar{\theta}$ given by the quasi-steady solution (3.17). However, the discrepancy is not due to ignoring the long-term slow variation of $\bar{\theta}$ in (3.17), but rather because we have not accounted for the development of $\bar{\theta}$ during the transient period. We need to calculate the transient in $\theta$ to higher order, so we write

$$
\theta=1+\operatorname{Gr} \theta_{1}+\mathrm{Gr}^{2} \theta_{2}
$$




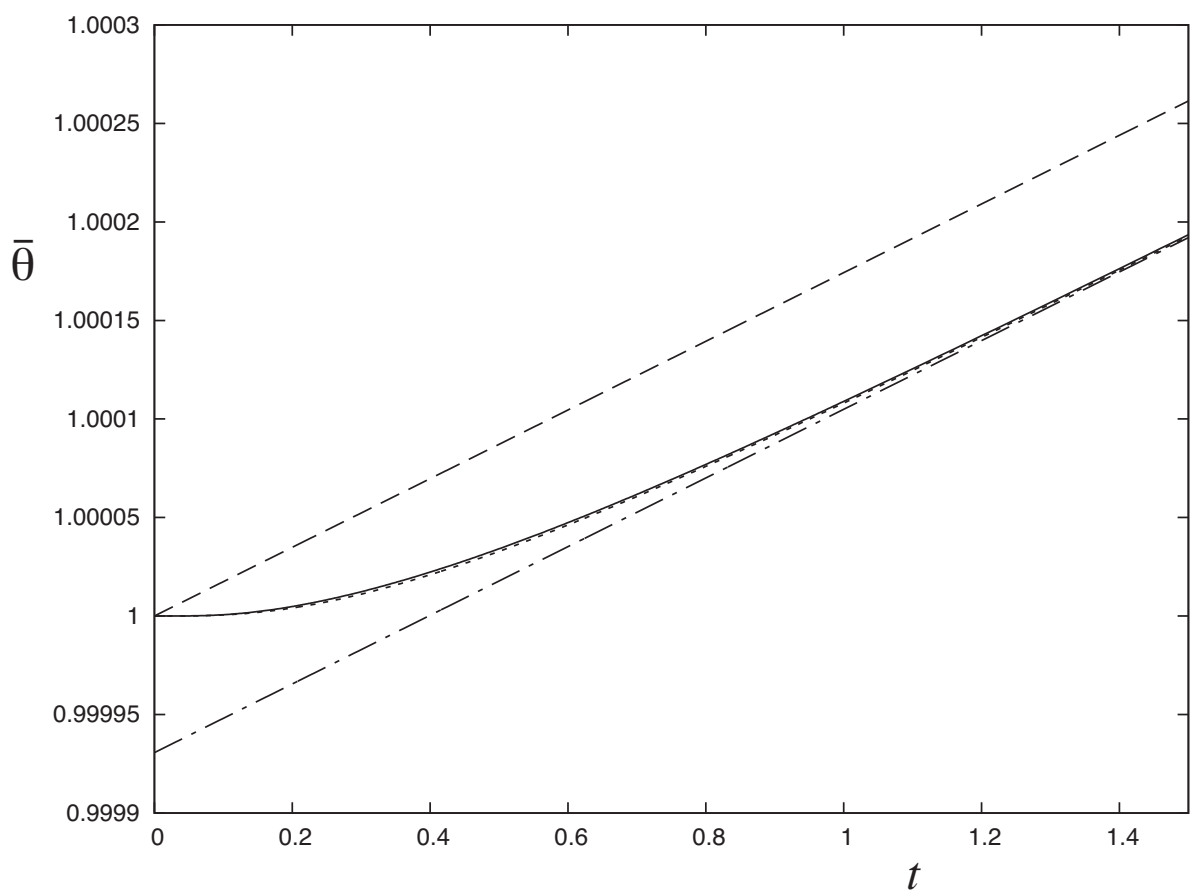

Figure 3: Development of $\bar{\theta}$ over the period $0<t<1.5$. Solid line: result of numerical computation. Dotted line: formula (A.3). Dashed line: formula (4.10). Dash-dotted line: formula (4.11). 
so that

$$
\frac{\mathrm{d} \bar{\theta}}{\mathrm{d} t}=\mathrm{Gr}^{2} \frac{\mathrm{d} \overline{\theta_{2}}}{\mathrm{~d} t}
$$

and we observe that $\bar{\theta}$ must satisfy $\mathrm{d} \bar{\theta} / \mathrm{d} t=0$ at $t=0$ as well as (3.16) at later times. The scalings that apply during the transient are

$$
w=\mathrm{Gr}^{1 / 2} w_{T}, \quad t=\mathrm{Gr}^{1 / 2} t_{T},
$$

and at $O\left(\mathrm{Gr}^{3 / 2}\right)$ in $(1.18)$ we obtain

$$
\frac{\partial \theta_{2}}{\partial t_{T}}-\operatorname{Pr}^{-1} \frac{\partial^{2} \theta_{2}}{\partial z^{2}}=\frac{\partial w_{T}}{\partial z} \theta_{1}-w_{T} \frac{\partial \theta_{1}}{\partial z}
$$

in which the forcing terms are obtained from the previously calculated formulae for $w$ and $\theta_{1}$ in $(4.2),(4.4),(3.10),(\mathrm{A} .1)$ and (A.2). Integrating (4.8) over the domain and applying the boundary conditions, we find that

$$
\frac{\mathrm{d} \overline{\theta_{2}}}{\mathrm{~d} t_{T}}=2 \int_{0}^{1} \frac{\partial w_{T}}{\partial z} \theta_{1} \mathrm{~d} z .
$$

We do not require the full solution of (4.8) for $\theta_{2}\left(z, t_{T}\right)$, since the variation of $\theta$ about its spatial mean is given to sufficient accuracy by our solution for $\theta_{1}$; in view of (4.6), our concern is to find $\overline{\theta_{2}}\left(t_{T}\right)$ in order to determine the temporal development of $\theta$. The right-hand side of (4.9) involves six terms, arising from steady and transient terms in $\partial w_{T} / \partial z$ multiplied by a steady and two transient terms in $\theta_{1}$ (see (4.4)). These are evaluated with the help of the symbolic manipulation package Mathematica; noting (4.6) and the initial condition $\bar{\theta}=1$ at $t_{T}=0$, we then integrate with respect to time to obtain $\bar{\theta}$. The final result, expressed in terms of the unscaled time variable $t$, is given as equation (A.3) in the Appendix and is shown as the dotted line in Figure 3, which is a close fit to the numerically computed solution shown as the solid line in Figure 3. The result (A.3) includes firstly the small-time approximation to the quasi-steady solution (3.17),

$$
1+\mathrm{Gr}^{3 / 2} \frac{\operatorname{Pr}}{181440} t
$$


which arises from the initial condition and the steady parts of $\partial w_{T} / \partial z$ and $\theta_{1}$ in the righthand side of (4.9), and is shown as a dashed line in Figure 3; the remaining terms in (A.3) constitute a displacement of $\bar{\theta}$ from the quasi-steady value. These terms approach a nonzero constant, uniform value as $t \rightarrow \infty$, so nonlinear interactions during the transient have a lasting effect on the temperature distribution; we may write

$$
\bar{\theta} \sim 1+\mathrm{Gr}^{3 / 2} \frac{\operatorname{Pr}}{181440} t+\mathrm{Gr}^{2} f(\mathrm{Pr})
$$

where the detailed form of $f(\mathrm{Pr})$ is given in the Appendix. The approximation (4.11) is shown by the dash-dotted line in Figure 3, and is valid while $\mathrm{Gr}^{1 / 2} \ll t \ll \mathrm{Gr}^{-3 / 2}$ (assuming also that $\mathrm{Pr}$ is of order unity). The condition $t \gg \mathrm{Gr}^{1 / 2}$ ensures that the transient has died away, while the condition $t \ll \mathrm{Gr}^{-3 / 2}$ ensures that the quasi-steady solution has not yet deviated significantly from linear variation; we may be rather lax in applying these conditions, since the factor $\pi^{2}$ in all the exponents in (A.3) means that the transients die away faster than the time-scale $\mathrm{Gr}^{1 / 2}$, while the factor 45360 in the quasi-steady solution (3.17) means that it deviates from linear growth more slowly than the time-scale $\mathrm{Gr}^{-3 / 2}$.

\section{Approach to blow-up}

The initial transient and the quasi-steady solutions display symmetry about the mid-point of the domain. There is also symmetry in the initial stages of the inviscid version of the flow (Kay, 1992), but this is soon broken due to the positive feedback between horizontal convergence (proportional to $-u$ ) and horizontal temperature gradient (proportional to $\theta$ ) near the upper boundary. As a result, the location $z_{w m}$ of the maximum of $|w|$ moves monotonically upwards in the inviscid flow. The behaviour is more complicated in the viscous case: as the quasi-steady regime breaks down, the symmetry in the $w$-profile is first broken by $z_{w m}$ moving upward a little, but as blow-up approaches the maximum of $|w|$ starts moving downward rather rapidly. Figure 4(a) shows profiles of $w$, normalised by its 
maximum absolute value $w_{\max }$, at several times: at $t=1420$ the profile is still to a very good approximation the symmetric quartic function (3.12); at $t=1437$ the maximum of $|w|$ is above the middle of the domain, but at two slightly later times $z_{w m}$ is seen to have moved rapidly downward. We may speculate that with a larger Grashof number, so that viscosity is less dominant, $z_{w m}$ would move further towards the upper boundary, i.e. more similarly to the inviscid case, before eventually moving downward.

The $\theta$ profile, normalised by its maximum value $\theta_{\max }$, is shown in Figure $4(\mathrm{~b})$ at the same times as the $w$ profiles. The $\theta$ profile is monotonic in $z$ in the quasi-steady regime (as it is in the inviscid flow (Grundy and Kay, 2003)), as seen at $t=1420$; however, at later times it develops an internal maximum at a location which moves downwards as blowup approaches. The explanation for this behaviour is as follows. In the viscous flow, the horizontal convergence is restrained by the no-slip condition at the upper boundary: $u=0$ at $z=1$ in viscous flow, whereas $|u|$ has its maximum at $z=1$ in the inviscid flow. Thus the growth in $\theta$ at the upper boundary is restrained. However, the convergence $-u$ does have a maximum at $z=(3+\sqrt{3}) / 6 \approx 0.7887$ in the quasi-steady viscous flow, so $\theta$ increases faster some distance below the upper boundary, creating the internal maximum. The downward flow $w$ then advects large values of $\theta$, and the consequent vorticity generation and convergence, towards the lower boundary. Eventually the flow intensifies to a level where, despite the nominally small value of Grashof number, viscosity ceases to be dominant except in boundary layers at the no-slip boundaries. In this regime, the downward flow keeps the lower boundary layer very thin, while the upper boundary layer remains too thick to be clearly distinguishable as a boundary layer: see Figure 6 below.

We now seek an asymptotic solution for the flow near blow-up, guided by the results of our numerical computations. We expect to find an outer solution, valid away from the boundaries and matched with boundary-layer solutions which apply near $z=0$ and $z=1$. 
(a)

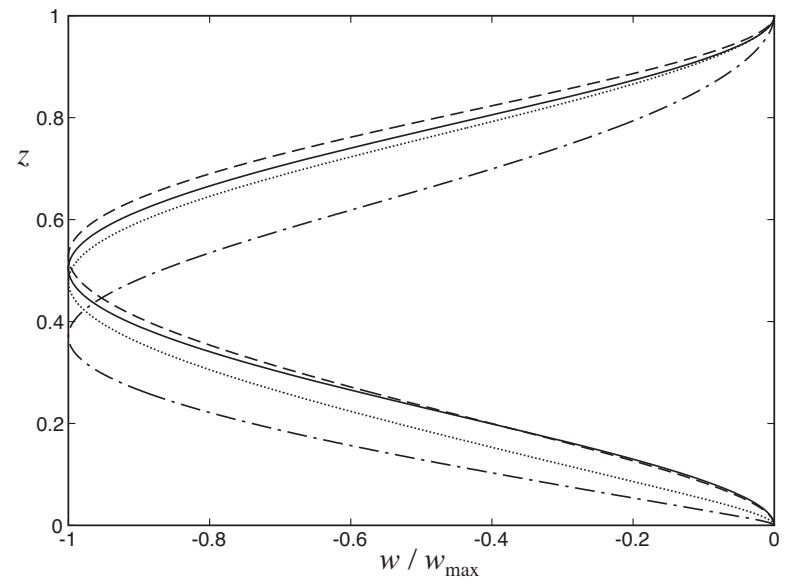

(b)

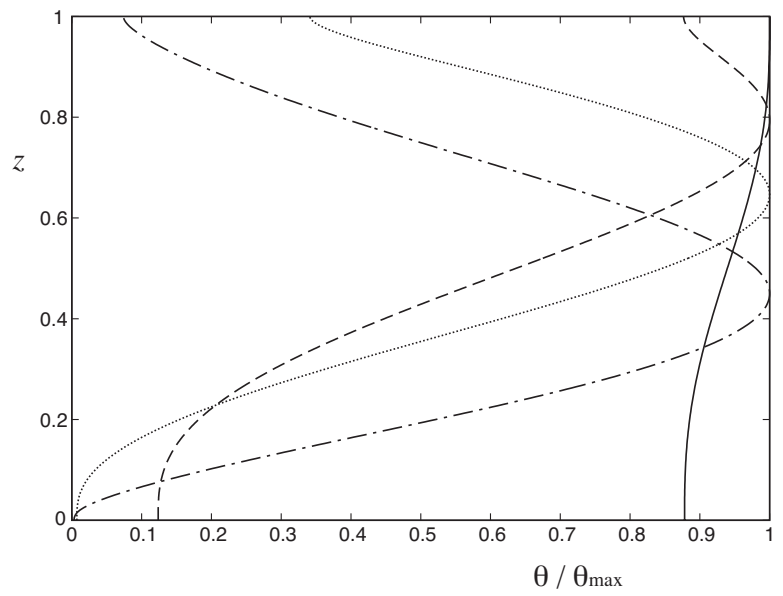

Figure 4: Numerically computed profiles of $w$, and $\theta$, normalised by $w_{\max }$ and $\theta_{\max }$ respectively, at times $t=1420$ (solid lines), $t=1437$ (dashed lines), $t=1437.06$ (dotted lines) and $t=1437.065$ (dash-dotted lines).

Assuming that blow-up does occur at some as yet undetermined time $t_{b}$, we set

$$
\tau=t_{b}-t
$$

and seek power-law behaviour

$$
\psi \sim \tau^{-\gamma} \quad \text { as } \tau \rightarrow 0
$$

for some $\gamma>0$, in our numerical solution; here $\psi$ represents any of the flow variables $w, \theta$, etc., evaluated at some specified point in the domain. Since $t_{b}$ and $\gamma$ are unknown, we plot $|\psi|^{-1 / \gamma}$ against $t$ with various trial values of $\gamma$ : when the correct value of $\gamma$ is selected, the plot will be a straight line, intersecting the abscissa at $t=t_{b}$. This methodology will be applied separately to the outer solution and each of the boundary layers.

\subsection{The outer solution}

We consider $w_{\max }$ and $\theta_{\max }$ to be representative of the behaviour of $w$ and $\theta$ in the outer flow, so we seek to determine blow-up power laws in the outer flow by plotting $w_{\max }^{-1 / \gamma}$ and $\theta_{\max }^{-1 / \gamma}$ 
(a)

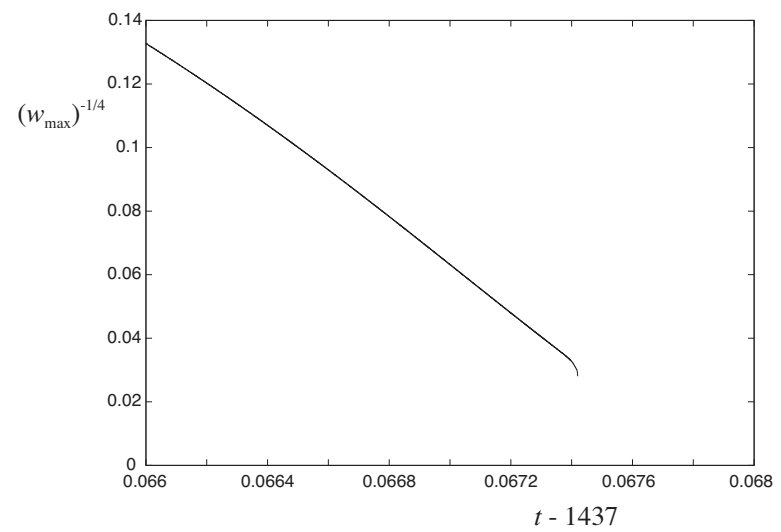

(b)

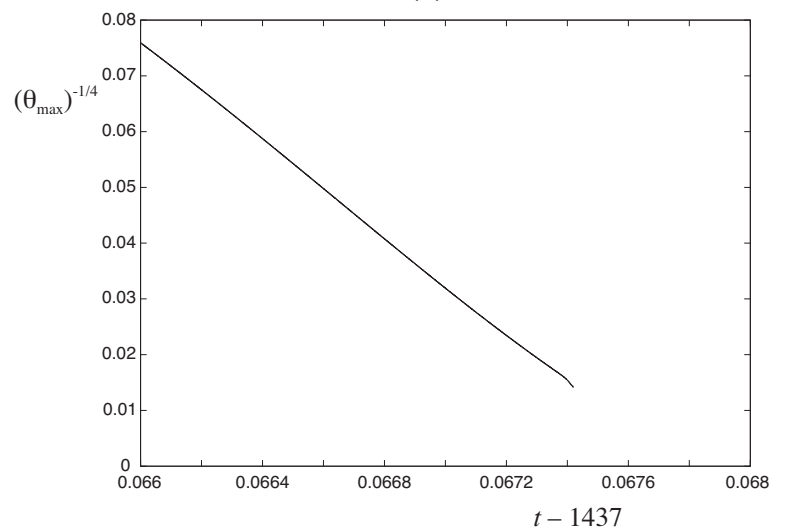

Figure 5: Numerically computed values of $w_{\max }^{-1 / 4}$ and $\theta_{\max }^{-1 / 4}$ as functions of time for a period before blow-up. As blow-up occurs shortly after $t=1437$, we have subtracted 1437 from the time in the abscissa annotations.

against time. The best straight lines appear to be obtained with $\gamma=4$ for both variables: see Figures 5 (a) and (b). Unfortunately we have not been able to confirm this analytically by the sort of argument used by Grundy and McLaughlin (1997) for the power law $\gamma=2$ in another blow-up problem involving stagnation-point similitude in a slab geometry. The blow-up time $t_{b}$ appears to be around 1437.0678, although we cannot be confident that the estimate is correct to the stated precision due to the possibility that small errors in the numerical computation may have accumulated over the long period of integration.

Assuming only that $w$ and $\theta$ obey the same power law, the quadratic buoyancy term in equation (1.17) is of the same order as the nonlinear advection terms. We define scaled variables $W$ and $\Theta$ according to

$$
w=-\frac{W}{\tau^{\gamma}}, \quad \theta=\frac{\Theta}{\tau^{\gamma}}
$$

so that equations (1.17) and (1.18) become

$$
\tau^{\gamma} \frac{\partial^{3} W}{\partial \tau \partial z^{2}}-\gamma \tau^{\gamma-1} \frac{\partial^{2} W}{\partial z^{2}}=\frac{\partial W}{\partial z} \frac{\partial^{2} W}{\partial z^{2}}-W \frac{\partial^{3} W}{\partial z^{3}}+\Theta^{2}-\mathrm{Gr}^{-1 / 2} \tau^{\gamma} \frac{\partial^{4} W}{\partial z^{4}}
$$




$$
\tau^{\gamma} \frac{\partial \Theta}{\partial \tau}-\gamma \tau^{\gamma-1} \Theta=\frac{\partial W}{\partial z} \Theta-W \frac{\partial \Theta}{\partial z}-\operatorname{Gr}^{-1 / 2} \operatorname{Pr}^{-1} \tau^{\gamma} \frac{\partial^{2} \Theta}{\partial z^{2}} .
$$

Provided that $\gamma>1$, which certainly appears correct, the leading-order balance is between the nonlinear advection and buoyancy terms in the vorticity equation, and between the two nonlinear advection terms in the temperature equation, when $\tau \ll \mathrm{Gr}^{1 / 2 \gamma}$. With these assumptions, we seek expansions in the form

$$
W=W_{0}(z)+\sum_{i=1}^{\infty} W_{i}(z) q_{i}(\tau), \quad \Theta=\Theta_{0}(z)+\sum_{i=0}^{\infty} \Theta_{i}(z) q_{i}(\tau),
$$

where $q_{1}(\tau)=o(1)$ and $q_{i+1}(\tau)=o\left(q_{i}(\tau)\right)$ as $\tau \rightarrow 0$. The leading-order balance in (5.15) and (5.16) is then

$$
\begin{aligned}
W_{0}^{\prime} W_{0}^{\prime \prime}-W_{0} W_{0}^{\prime \prime \prime}+\Theta_{0}^{2} & =0 \\
W_{0}^{\prime} \Theta_{0}-W_{0} \Theta_{0}^{\prime} & =0
\end{aligned}
$$

in which primes indicate derivatives with respect to $z$. Equation (5.19) may be integrated immediately to yield

$$
\Theta_{0}=c W_{0}
$$

in which we shall see that the "constant" $c$ may vary slowly in time. Using (5.20) to substitute for $\Theta_{0}$, we may write (5.18) in the form

$$
-W_{0}^{2} \frac{\mathrm{d}}{\mathrm{d} z}\left(\frac{W_{0}^{\prime \prime}}{W_{0}}\right)+c^{2} W_{0}^{2}=0 .
$$

This has a first integral

$$
W_{0}^{\prime \prime}=\left(c^{2} z+b\right) W_{0}
$$

where $b$ is a constant of integration. Equation (5.22) has a general solution in terms of Airy functions,

$$
W_{0}=\alpha \operatorname{Ai}\left(c^{2 / 3} z+c^{-4 / 3} b\right)+\beta \operatorname{Bi}\left(c^{2 / 3} z+c^{-4 / 3} b\right)
$$


(a)

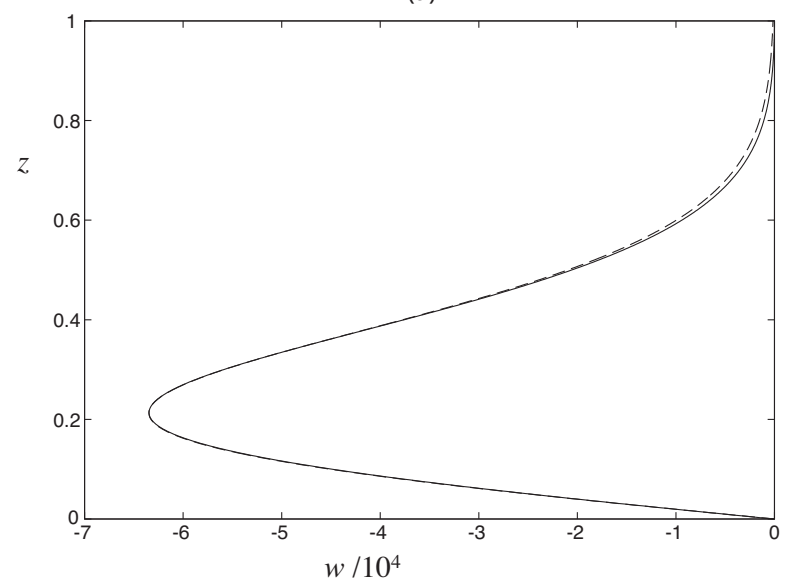

(b)

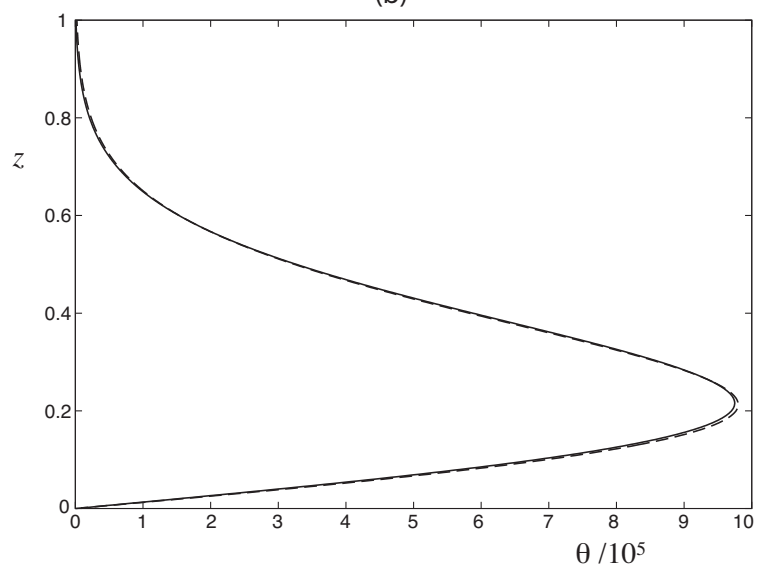

Figure 6: Computed profiles and Airy function approximations to (a) $w$ and (b) $\theta$, at $t=$ 1437.067. Solid lines are computed profiles. The magnitude of the computed maximum of $w$ was used to determine the multiplier $1.185 \times 10^{5}$ in $(5.24)$, while its location yielded the coefficient 6.2 in the argument of the Airy function. The dashed curve in (a) is the function (5.24), and the dashed curve in (b) is this function multiplied by $-6.2^{3 / 2}$.

in which there are four undetermined coefficients. These should, in principle, be determined not by the four boundary conditions on $w$ but by matching with solutions in the boundary layers. However, we seek guidance from the numerical solution. Figure 6(a) shows that at time $t=1437.067$, very close to the estimated blow-up time, the profile of $w$ is an excellent fit to

$$
-1.185 \times 10^{5} \mathrm{Ai}\left(6.2 z+a_{1}\right)
$$

where $a_{1} \approx-2.338$ is the first zero of the Airy function Ai. Thus the coefficients in (5.23) appear to be

$$
\beta=0, \quad c \approx 6.2^{3 / 2}, \quad b=a_{1} c^{4 / 3}, \quad \alpha \approx-1.185 \times 10^{5}\left(t_{b}-1437.067\right)^{\gamma} .
$$

According to (5.14) and (5.20), we should have $\theta \approx-c w$ at leading order. Multiplying the function (5.24) by $-c$, using the estimate of $c$ in (5.25), does indeed give a good fit 


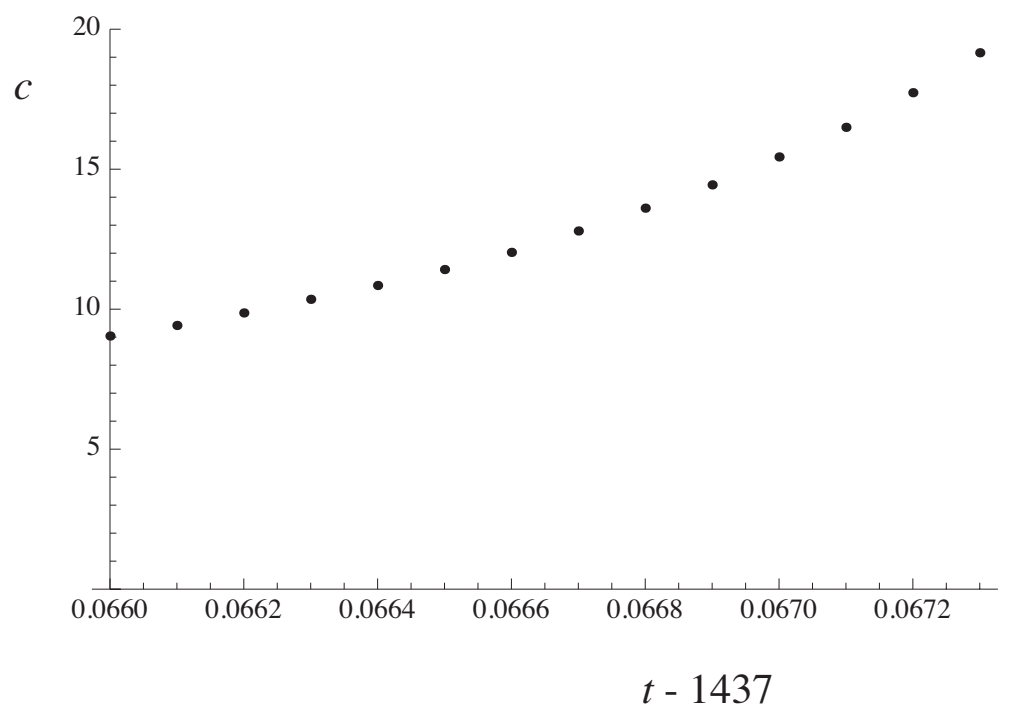

Figure 7: Values of $c$ estimated from fitting functions of form $\alpha \operatorname{Ai}\left(c^{2 / 3} z+a_{1}\right)$ to computed profiles of $w$ at various times shortly before blow-up.

to the computed $\theta$-profile, as shown in Figure 6(b). However, the value of $c$ required for the best fit of the $w$-profile to the Airy function increases as blow-up approaches, as shown in Figure 7. The plotted points fit a curve of form $c \propto\left(t_{b}-t\right)^{-1}$ fairly well, but with $t_{b} \approx 1437.0685$, substantially later than the blow-up time estimated from Figure 5. We might propose a multiple-scales solution, with the magnitude of $W$ and $\Theta$ depending on the fast time variable $\tau$ while $c$ varies as a function of a slow time variable $s$; but it seems more likely that the behaviour of $c$ is controlled by the boundary layer near $z=1$.

We cannot yet be certain about the value of $\gamma$ or the form of the functions $q_{i}(\tau)$ in the expansions (5.17). Nevertheless, it is possible to make some progress regarding higher-order terms in these expansions. Expanding (5.15) and (5.16), using (5.20) to eliminate $\Theta_{0}$ and (5.22) to eliminate $W_{0}^{\prime \prime}$ and $W_{0}^{\prime \prime \prime}$, we find that each $W_{i}(z)$ and $\Theta_{i}(z)$ satisfies linear equations of form

$$
W_{0} W_{i}^{\prime \prime \prime}-W_{0}^{\prime} W_{i}^{\prime \prime}-\left(c^{2} z+b\right) W_{0} W_{i}^{\prime}+\left(c^{2} z+b\right) W_{0}^{\prime} W_{i}+c^{2} W_{0} W_{i}
$$




$$
\begin{aligned}
& =2 c W_{0} \Theta_{i}+f(z) \\
W_{0} \Theta_{i}^{\prime}-W_{0}^{\prime} \Theta_{i}-c W_{0} W_{i}^{\prime}+c W_{0}^{\prime} W_{i} & =g(z)
\end{aligned}
$$

where $f(z)$ and $g(z)$ depend only on lower-order terms already calculated. Equation (5.27) has the solution

$$
\Theta_{i}=c W_{i}+W_{0} \int \frac{g(z)}{W_{0}^{2}} \mathrm{~d} z .
$$

This may be substituted into (5.26), which can then be written

$$
\frac{\mathrm{d}}{\mathrm{d} z}\left(\frac{W_{i}^{\prime \prime}}{W_{0}}-\left(c^{2} z+b\right) \frac{W_{i}}{W_{0}}\right)=F(z)
$$

where

$$
F(z)=\frac{f(z)}{W_{0}^{2}}+2 c \int \frac{g(z)}{W_{0}^{2}} \mathrm{~d} z .
$$

The general solution of $(5.29)$ is

$$
W_{i}=W_{0} \int \frac{1}{W_{0}^{2}}\left(\int W_{0}^{2}\left(\int F(z) \mathrm{d} z\right) \mathrm{d} z\right) \mathrm{d} z
$$

in which each of the four indefinite integrals in (5.31) and (5.30) implies the presence of a constant of integration.

The expression (5.24) satisfies the boundary condition $w=0$ at $z=0$, so we have a classical viscous boundary layer there, with the outer flow satisfying the rigid-wall condition but not the no-slip condition. The temperature has a boundary layer of the same thickness (in the case with $\operatorname{Pr}=1$ ) in order to satisfy the insulating boundary condition. In contrast, since the Airy function decays exponentially at large positive values of its argument, a solution like (5.24) cannot satisfy either of the boundary conditions at $z=1$; hence it is near the upper boundary that the discrepancy between the numerical solution and the Airy function approximation is greatest in Figure 6. 


\subsection{The lower boundary layer}

The numerical solution shows the thickness of the boundary layer adjacent to the wall at $z=0$ decreasing as blow-up approaches. This suggests the use of a scaled coordinate

$$
\zeta=\frac{z}{\tau^{\delta}}
$$

for some $\delta>0$, together with scaled dependent variables

$$
v=\frac{w}{\tau^{\epsilon}}, \quad \phi=\frac{\theta}{\tau^{\kappa}} .
$$

In terms of these boundary-layer variables, equations (1.17) and (1.18) become

$$
\begin{aligned}
-\tau^{\delta-\epsilon} \frac{\partial^{3} v}{\partial \tau \partial \zeta^{2}}+\delta \tau^{\delta-\epsilon-1} \zeta \frac{\partial^{3} v}{\partial \zeta^{3}}-(\epsilon-2 \delta) \tau^{\delta-\epsilon-1} \frac{\partial^{2} v}{\partial \zeta^{2}}= & \frac{\partial v}{\partial \zeta} \frac{\partial^{2} v}{\partial \zeta^{2}}-v \frac{\partial^{3} v}{\partial \zeta^{3}}+\tau^{2 \kappa-2 \epsilon+3 \delta} \phi^{2} \\
& +\mathrm{Gr}^{-1 / 2} \tau^{-\delta-\epsilon} \frac{\partial^{4} v}{\partial \zeta^{4}} \\
-\tau^{\delta-\epsilon} \frac{\partial \phi}{\partial \tau}+\delta \tau^{\delta-\epsilon-1} \zeta \frac{\partial \phi}{\partial \zeta}-\kappa \tau^{\delta-\epsilon-1} \phi= & \frac{\partial v}{\partial \zeta} \phi-v \frac{\partial \phi}{\partial \zeta} \\
& +\operatorname{Gr}^{-1 / 2} \operatorname{Pr}^{-1} \tau^{-\delta-\epsilon} \frac{\partial^{2} \phi}{\partial \zeta^{2}} .
\end{aligned}
$$

The viscous term in (5.34) and the diffusive term in (5.35) must be of leading order within the boundary layer, so

$$
\epsilon=-\delta
$$

Near $z=0$, the outer solution (5.23) has the form

$$
W \sim A_{0} z,
$$

where

$$
A_{0}=\alpha c^{2 / 3} \operatorname{Ai}^{\prime}\left(a_{1}\right) .
$$

In terms of boundary-layer variables, (5.37) becomes

$$
-\tau^{\gamma+\epsilon} v \sim A_{0} \zeta \tau^{\delta},
$$


so matching requires

$$
\gamma+\epsilon=\delta
$$

Together with (5.36), this yields

$$
\delta=\frac{\gamma}{2}, \quad \epsilon=-\frac{\gamma}{2}
$$

Since the outer solution also has

$$
\Theta \sim c A_{0} z
$$

near $z=0$, a similar matching argument gives

$$
\kappa=-\frac{\gamma}{2}
$$

If $\gamma=4$ as suggested by Figure 5, we have

$$
\delta=2, \quad \epsilon=\kappa=-2
$$

For comparison with the computational results, we observe that (5.44) implies that

$$
\omega\left(=-\frac{\partial^{2} w}{\partial z^{2}}\right) \propto \tau^{-6}
$$

in the boundary layer. This is supported by Figure 8 which is a plot of $\left|\omega_{l}\right|^{-1 / 6}$ against time, where the subscript $l$ indicates values at the lower boundary $z=0$. Note that the computations do not resolve the boundary layer at the times in this plot, so such good agreement may be surprising; however, the numerical algorithm integrates $\omega$ forward in time, with the boundary conditions on $w$ and $u(=-\partial w / \partial z)$ only being applied at each time-step after $\omega$ has been computed. In contrast, the boundary condition on $\partial \theta / \partial z$ is applied as $\theta$ is integrated in time, so non-resolution of the boundary layer renders values of $\theta_{l}$ invalid; indeed, plots of computed $\theta_{l}$ (not shown here) show a rather irregular dependence on $\tau$ as blow-up approaches. 


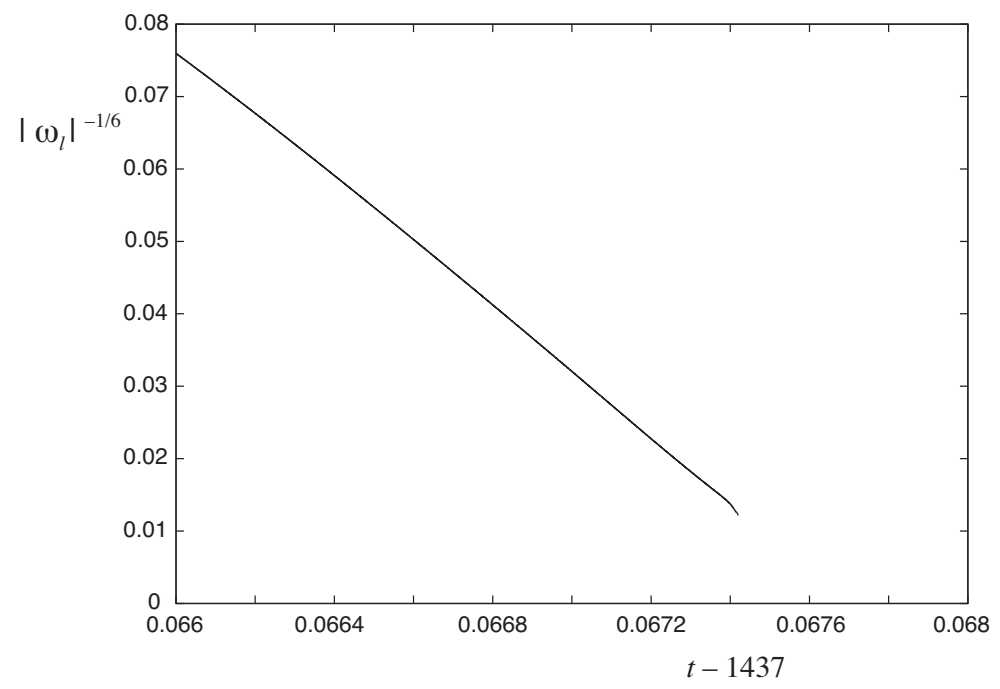

Figure 8: Numerically computed values of $\left|\omega_{l}\right|^{-1 / 6}$ as a function of time for a period before blow-up.

The buoyancy term in (5.34) is now $\tau^{3 \delta} \phi^{2}$ so, since $\delta>0$, the temperature behaves as a passive tracer (at leading order) in the lower boundary layer. The leading-order boundarylayer flow is then given by

$$
v_{0}^{\prime} v_{0}^{\prime \prime}-v_{0} v_{0}^{\prime \prime \prime}+\mathrm{Gr}^{-1 / 2} v_{0}^{\prime \prime \prime \prime}=0
$$

where primes indicate differentiation with respect to $\zeta$, and $v$ has been expanded as

$$
v(\zeta, \tau)=v_{0}(\zeta)+\text { terms of higher order in } \tau \text {. }
$$

The wall boundary conditions (1.19) become

$$
v_{0}(0)=0, \quad v_{0}^{\prime}(0)=0
$$

while matching with the outer flow (see equations $(5.37)-(5.40)$ ) yields

$$
v_{0}^{\prime}(\zeta) \rightarrow-A_{0} \text { as } \zeta \rightarrow \infty
$$


A first integral of (5.46) is

$$
v_{0}^{\prime 2}-v_{0} v_{0}^{\prime \prime}+\mathrm{Gr}^{-1 / 2} v_{0}^{\prime \prime \prime}=K
$$

in which the constant $K$ is determined by noting that $v^{\prime \prime}(\zeta) \rightarrow 0$ and $v^{\prime \prime \prime}(\zeta) \rightarrow 0$ as $\zeta \rightarrow \infty$, so that (5.49) yields

$$
K=A_{0}^{2}
$$

The third-order boundary-value problem (5.48) - (5.51) can in principle be solved numerically, but $A_{0}$ involves quantities arising in the outer solution which are as yet undetermined. Once $v_{0}$ is found, it remains to solve the linear boundary value problem for $\phi_{0}$ (the leading term in an expansion of $\phi$ analogous to (5.47)):

$$
\begin{gathered}
v_{0}^{\prime} \phi_{0}-v \phi_{0}^{\prime}+\mathrm{Gr}^{-1 / 2} \operatorname{Pr}^{-1} \phi_{0}^{\prime \prime}=0, \\
\phi_{0}^{\prime}(0)=0, \quad \phi_{0}^{\prime}(\zeta) \rightarrow c A_{0} \text { as } \zeta \rightarrow \infty .
\end{gathered}
$$

\subsection{The upper boundary layer}

We again seek a solution in terms of scaled variables near $z=1$ : writing

$$
\eta=\frac{1-z}{\tau^{\mu}}, \quad V=\frac{w}{\tau^{\rho}}, \quad \Phi=\frac{\theta}{\tau^{\sigma}}
$$

we obtain equations of rather similar form to (5.34) and (5.35). The requirement for viscous and diffusive terms to be of leading order near a wall then yields

$$
\rho=-\mu
$$

The outer Airy function solution does not yield a simple matching function comparable to (5.37) near $z=1$, so we turn straight to the numerical solution. Figure 9 suggests that

$\omega_{u} \propto \tau^{-3 / 2}$ and $\theta_{u} \propto \tau^{-3 / 4}$ (where the subscript $u$ indicates values at the upper wall), although the apparent blow-up time is noticeably later than that estimated for the outer flow. Ignoring this discrepancy, we therefore have

$$
\rho-2 \mu=-\frac{3}{2}, \quad \sigma=-\frac{3}{4},
$$



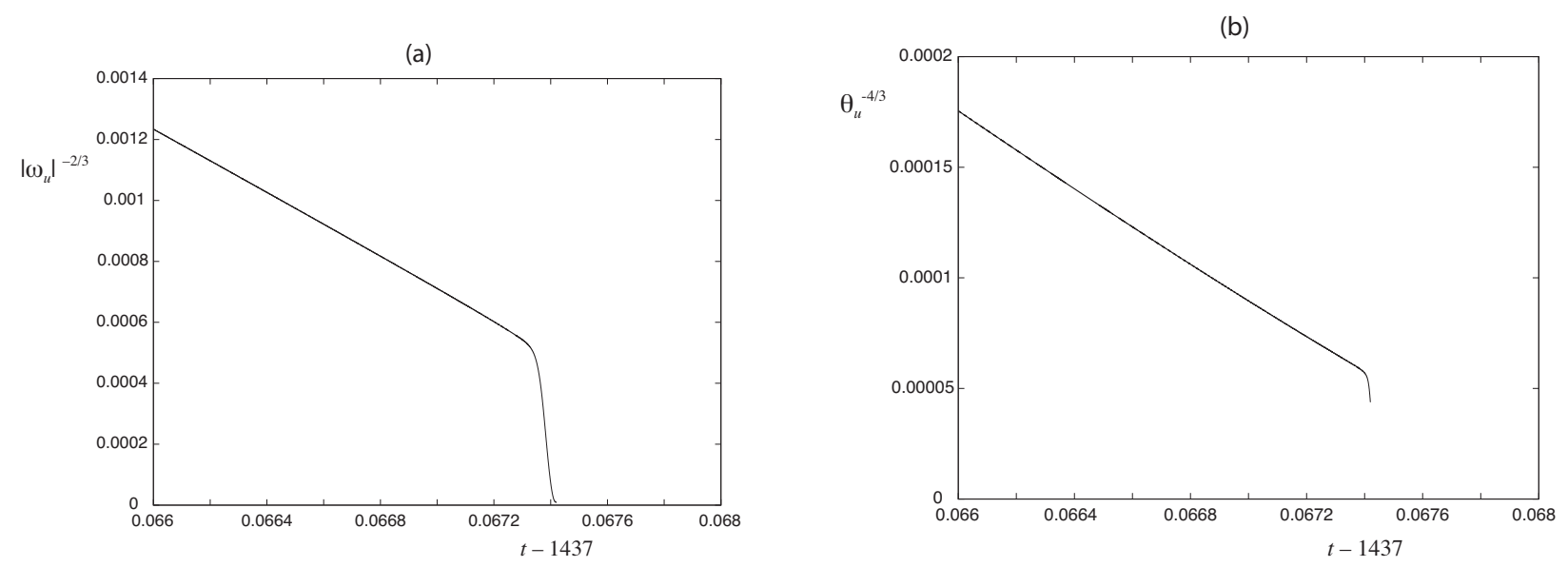

Figure 9: Numerically computed values of (a) $\left|\omega_{u}\right|^{-2 / 3}$ and (b) $\theta_{u}^{-4 / 3}$ as functions of time for a period before blow-up. The failure of the computational algorithm around $t=1437.0674$ is particularly obvious in these plots.

in which the first equation combines with (5.55) to yield

$$
\mu=\frac{1}{2}, \quad \rho=-\frac{1}{2} .
$$

The scalings $w \propto \tau^{-1 / 2}$ and $\theta \propto \tau^{-3 / 4}$ are just those in the blow-up of the quasi-steady solution, (3.12) and (3.15) with (3.17), but now confined to a layer of width $\propto \tau^{1 / 2}$, sufficiently broad to be resolved by the numerical algorithm at times shown in Figure 9. With these scalings the vorticity and temperature equations (1.17) and (1.18) become

$$
\begin{aligned}
\frac{1}{2} \eta \frac{\partial^{3} V}{\partial \eta^{3}}+\frac{3}{2} \frac{\partial^{2} V}{\partial \eta^{2}}-\tau \frac{\partial^{3} V}{\partial \tau \partial \eta^{2}} & =-\frac{\partial V}{\partial \eta} \frac{\partial^{2} V}{\partial \eta^{2}}+V \frac{\partial^{3} V}{\partial \eta^{3}}+\tau \Phi^{2}+\operatorname{Gr}^{-1 / 2} \frac{\partial^{4} V}{\partial \eta^{4}} \\
\frac{1}{2} \eta \frac{\partial \Phi}{\partial \eta}+\frac{3}{4} \Phi-\tau \frac{\partial \Phi}{\partial \eta} & =-\frac{\partial V}{\partial \eta} \Phi+V \frac{\partial \Phi}{\partial \eta}+\operatorname{Gr}^{-1 / 2} \operatorname{Pr}^{-1} \frac{\partial^{2} \Phi}{\partial \eta^{2}}
\end{aligned}
$$

in which all terms except the buoyancy term and the $\tau$-derivatives are of leading order. Thus even the leading-order problem seems intractable, and in any case we do not have any matching conditions with the outer flow. 


\section{Discussion}

The flow we have studied develops in three phases, in a fast-slow-fast sequence like the movements of a classical concerto:-

1. Allegro: The transient, in which the flow adjusts from the initial conditions to a balance between viscous and buoyancy forces.

2. Adagio: The quasi-steady regime, in which the buoyancy-viscous balance slowly intensifies the flow.

3. Presto: The final approach to blow-up, in which nonlinear advection terms become dominant, balancing buoyancy in the outer flow but balancing viscous forces in the boundary layers.

For the case of small Grashof number and $O(1)$ Prandtl number the asymptotic structure of the flow and temperature distribution has been fully determined for the first two stages, but the final stage has been more problematic. The dominance of advection in this stage causes $w$ and $\theta$ to adopt the same Airy function profile, but important details of this profile remain undetermined. In particular, the variation of the parameter $c$ corresponds to the peak becoming narrower and closer to the lower boundary as blow-up approaches, but we have not been able to derive any theory about this behaviour. We suspect that it may be somehow controlled by the flow in the upper boundary layer, which retains some features of the quasi-steady regime throughout the approach to blow-up, but no analytical matching of the outer and upper-boundary flows has been possible.

We have not proved rigorously that blow-up occurs, but our combination of numerical computation and asymptotic analysis has provide strong evidence for blow-up. In particular, the numerical solution fits an Airy function very well in the final stage of the flow, and the Airy function solution for the outer flow was derived on the basis of a blow-up scaling (a 
positive value of $\gamma$ in (5.14)). A possible approach to proving blow-up would be to use the quasi-steady solution as the basis for finding a rigorous lower bound for some flow parameter as a function of time: for instance, a function related to the quasi-steady approximation (3.17) for $\bar{\theta}$ might provide a rigorous lower bound for $\bar{\theta}$ which blows up.

Proving blow-up in the present case would obviously not be a major contribution to the debate on the regularity of solutions of the Navier-Stokes equations, since the initial density configuration has infinite potential energy. Nevertheless, infinite-energy solutions of Euler and Navier-Stokes are of interest (see, e.g. Ohkitani and Gibbon (2000)), and a feature of particular interest in the present case is that blow-up apparently occurs for arbitrarily large values of the viscosity, albeit after an arbitrarily long time. The equations for flow in an infinite slab with a quadratic horizontal density distribution have previously been proved to blow up when the flow is inviscid in an inertial frame (Jacqmin, 1991), while strong evidence

for blow-up has been obtained for inviscid flow in a rotating frame (Kay, 2004) and now for viscous flow in an inertial frame. Both coriolis and viscous effects delay the blow-up, and a possible subject for further investigation would be whether the combination of these effects could prevent blow-up entirely.

\section{Acknowledgements}

I thank Bob Grundy and Ray McLaughlin for useful discussions in the early stages of this work. 


\section{Appendix: details of initial transient}

The terms $\theta_{t s}$ and $\theta_{t t}$ in (4.4) are respectively the solutions to (4.3) with the forcing $\partial w / \partial z$

calculated from the steady and transient parts of $w$ in (4.2). We find

$$
\begin{aligned}
\theta_{t s}=\operatorname{Pr} \sum_{m=0}^{\infty} & \left(\frac{4}{(2 m+1)^{6} \pi^{6}}-\frac{1}{3(2 m+1)^{4} \pi^{4}}\right) \\
& \times \cos \{(2 m+1) \pi z\} \exp \left\{-(2 m+1)^{2} \pi^{2} \operatorname{Pr}^{-1} \mathrm{Gr}^{-1 / 2} t\right\}
\end{aligned}
$$

and

$$
\begin{aligned}
\theta_{t t}=\operatorname{Pr} \sum_{m=0}^{\infty} & \sum_{q=0}^{\infty} \frac{2 \cos \{(2 m+1) \pi z\}}{(2 q+1)^{2}\left((4 q+2)^{2}-(2 m+1)^{2}\right)\left((2 m+1)^{2}-\operatorname{Pr}(4 q+2)^{2}\right) \pi^{6}} \\
& \times\left(\exp \left\{-(4 q+2)^{2} \pi^{2} \mathrm{Gr}^{-1 / 2} t\right\}-\exp \left\{-(2 m+1)^{2} \pi^{2} \operatorname{Pr}^{-1} \mathrm{Gr}^{-1 / 2} t\right\}\right)
\end{aligned}
$$

Evaluation of the right-hand side of (4.9) and integration with the initial condition $\bar{\theta}=1$ at $\tau=0$ yields

$$
\begin{aligned}
\bar{\theta} \sim 1 & +\mathrm{Gr}^{3 / 2} \frac{\operatorname{Pr}}{181440} t \\
& -\mathrm{Gr}^{2} \frac{\operatorname{Pr}}{256} \sum_{p=0}^{\infty}\left(\frac{1}{(2 p+1)^{10} \pi^{10}}+\frac{1}{45(2 p+1)^{6} \pi^{6}}\right)\left(1-\exp \left\{-(4 p+2)^{2} \pi^{2} \mathrm{Gr}^{-1 / 2} t\right\}\right) \\
& -\mathrm{Gr}^{2} \operatorname{Pr}^{2} \sum_{m=0}^{\infty} \frac{1}{(2 m+1)^{8} \pi^{8}}\left(\frac{1}{3}-\frac{4}{(2 m+1)^{2} \pi^{2}}\right)^{2}\left(1-\exp \left\{-(2 m+1)^{2} \pi^{2} \operatorname{Pr}^{-1} \mathrm{Gr}^{-1 / 2} t\right\}\right) \\
& +2 \mathrm{Gr}^{2} \operatorname{Pr} \sum_{p=0}^{\infty} \sum_{m=0}^{\infty} \frac{1}{(2 p+1)^{2}(2 m+1)^{4}\left\{(2 m+1)^{2}-(4 p+2)^{2}\right\}\left\{\operatorname{Pr}^{-1}(2 m+1)^{2}+(4 p+2)^{2}\right\} \pi^{10}} \\
& \times\left(\frac{1}{3}-\frac{4}{(2 m+1)^{2} \pi^{2}}\right)\left(1-\exp \left\{-\left\{\operatorname{Pr}^{-1}(2 m+1)^{2}+(4 p+2)^{2}\right\} \pi^{2} \mathrm{Gr}^{-1 / 2} t\right\}\right) \\
& -2 \mathrm{Gr}^{2} \sum_{q=0}^{\infty} \sum_{m=0}^{\infty} \frac{1}{(2 q+1)^{2}(2 m+1)^{2}\left\{(4 q+2)^{2}-(2 m+1)^{2}\right\}\left\{\operatorname{Pr}^{-1}(2 m+1)^{2}-(4 q+2)^{2}\right\} \pi^{10}} \\
& \times\left(\frac{1}{3}-\frac{4}{(2 m+1)^{2} \pi^{2}}\right)
\end{aligned}
$$




$$
\begin{gathered}
\times\left\{\frac{1-\exp \left\{-(4 q+2)^{2} \pi^{2} \mathrm{Gr}^{-1 / 2} t\right\}}{(4 q+2)^{2}}-\frac{1-\exp \left\{-(2 m+1)^{2} \pi^{2} \operatorname{Pr}^{-1} \mathrm{Gr}^{-1 / 2} t\right\}}{(2 m+1)^{2} \operatorname{Pr}^{-1}}\right\} \\
-4 \mathrm{Gr}^{2} \sum_{p=0}^{\infty} \sum_{q=0}^{\infty} \sum_{m=0}^{\infty} \frac{1}{(2 p+1)^{2}(2 q+1)^{2}\left\{(2 m+1)^{2}-(4 p+2)^{2}\right\}\left\{(4 q+2)^{2}-(2 m+1)^{2}\right\} \pi^{12}} \\
\times \frac{1}{\operatorname{Pr}^{-1}(2 m+1)^{2}-(4 q+2)^{2}}\left\{\frac{1-\exp \left\{-\left\{(4 p+2)^{2}+(4 q+2)^{2}\right\} \pi^{2} \mathrm{Gr}^{-1 / 2} t\right\}}{(4 p+2)^{2}+(4 q+2)^{2}}\right. \\
\left.-\frac{1-\exp \left\{-\left\{\operatorname{Pr}^{-1}(2 m+1)^{2}+(4 p+2)^{2}\right\} \pi^{2} \mathrm{Gr}^{-1 / 2} t\right\}}{\operatorname{Pr}^{-1}(2 m+1)^{2}+(4 p+2)^{2}}\right\} .
\end{gathered}
$$

Taking the limit as $t \rightarrow \infty$ yields

$$
\bar{\theta} \sim 1+\mathrm{Gr}^{3 / 2} \frac{\mathrm{Pr}}{181440} t+\mathrm{Gr}^{2} f(\operatorname{Pr})
$$

in which

$$
\begin{aligned}
f(\operatorname{Pr})=- & \frac{17}{2^{19} \times 3^{2} \times 5 \times 7}-\frac{491}{2^{17} \times 3^{4} \times 5^{2} \times 7} \operatorname{Pr}-\frac{131}{2^{9} \times 3^{5} \times 5^{2} \times 7 \times 11} \operatorname{Pr}^{2} \\
& +\frac{-310+1220 \operatorname{Pr}+1685 \operatorname{Pr}^{2}+7724 \operatorname{Pr}^{3}-28160 \operatorname{Pr}^{4}}{2^{20} \times 3^{4} \times 5^{2} \times 7 \operatorname{Pr}(1+\operatorname{Pr})} \\
& -\frac{\operatorname{Pr}^{7 / 2}}{1+\operatorname{Pr}} \sum_{m=0}^{\infty} \frac{1}{(1+2 m)^{9} \pi^{9}}\left(\frac{1}{3}-\frac{4}{(2 m+1)^{2} \pi^{2}}\right) \tanh \left\{\frac{(2 m+1) \pi}{4 \operatorname{Pr}^{1 / 2}}\right\} \\
& +\frac{1}{1024 \operatorname{Pr}^{1 / 2}(1+\operatorname{Pr})} \sum_{p=0}^{\infty} \frac{\tanh \left\{(2 p+1) \pi \operatorname{Pr}^{1 / 2}\right\}}{(2 p+1)^{9} \pi^{9}} \\
& +\frac{1}{1024(\operatorname{Pr}-1)} \sum_{p=0}^{\infty} \frac{1}{(2 p+1)^{10} \pi^{10}}\left(\operatorname{Pr} \operatorname{sech}\{(2 p+1) \pi\}-\frac{2}{\operatorname{Pr}(1+\operatorname{Pr})} \operatorname{sech}\left\{(2 p+1) \pi \operatorname{Pr}^{1 / 2}\right\}\right. \\
& \left.+\tanh \left\{\frac{(2 p+1) \pi}{2}\right\}\left(\operatorname{Pr} \tanh \{(2 p+1) \pi\}-\frac{2 \operatorname{Pr}^{1 / 2}}{1+\operatorname{Pr}} \tanh \left\{(2 p+1) \pi \operatorname{Pr}^{1 / 2}\right\}\right)\right) .(\operatorname{A.5})
\end{aligned}
$$

The quantity $f(\operatorname{Pr})$ is negative for all $\operatorname{Pr}>0$, and a log-log plot of $-f(\operatorname{Pr})$ is shown in Figure 10. Since (A.5) requires l'Hôpital's rule to evaluate when $\operatorname{Pr}=1$, we note here the 


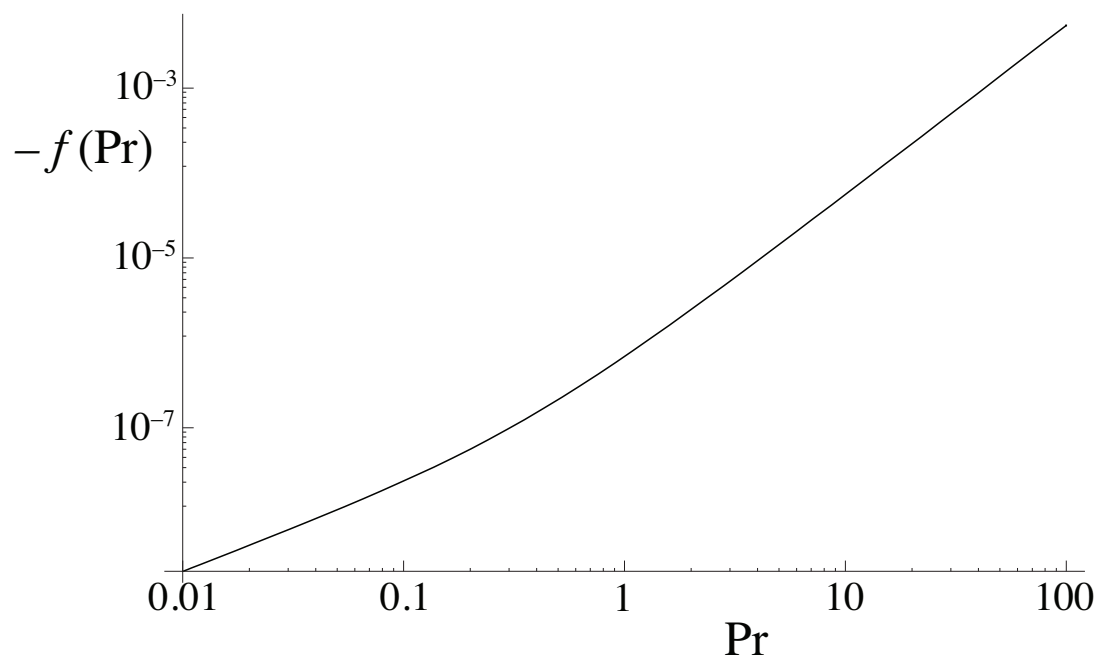

Figure 10: The function $f(\operatorname{Pr})$ as given by (A.5)

result

$$
\begin{aligned}
& f(1)=- \frac{1485557}{2^{21} \times 3^{5} \times 5^{2} \times 7 \times 11}-\frac{1}{2} \sum_{m=0}^{\infty} \frac{1}{(1+2 m)^{9} \pi^{9}}\left(\frac{1}{3}-\frac{4}{(2 m+1)^{2} \pi^{2}}\right) \tanh \left\{\frac{(2 m+1) \pi}{4}\right\} \\
&+\frac{1}{2048} \sum_{p=0}^{\infty} \frac{1}{(2 p+1)^{9} \pi^{9}}(\tanh \{(2 p+1) \pi\}(1+\operatorname{sech}\{(2 p+1) \pi\}) \\
&\left.\quad-\operatorname{sech}^{2}\{(2 p+1) \pi\} \tanh \left\{\frac{(2 p+1) \pi}{2}\right\}\right) \\
& \quad+\frac{1}{2048} \sum_{p=0}^{\infty} \frac{1}{(2 p+1)^{10} \pi^{10}}\left(5 \operatorname{sech}\{(2 p+1) \pi\}+2 \tanh \{(2 p+1) \pi\} \tanh \left\{\frac{(2 p+1) \pi}{2}\right\}\right) \\
& \approx- 6.937 \times 10^{-7} .
\end{aligned}
$$

Furthermore, as $\operatorname{Pr} \rightarrow 0$,

$$
\begin{aligned}
f(\operatorname{Pr}) \sim & \left\{-\frac{3733}{2^{20} \times 3^{4} \times 5^{2} \times 7}+\frac{1}{512} \sum_{p=0}^{\infty} \frac{1}{(2 p+1)^{9} \pi^{9}} \tanh \left\{\frac{(2 p+1) \pi}{2}\right\}\right. \\
& \left.-\frac{1}{1024} \sum_{p=0}^{\infty} \frac{1}{(2 p+1)^{10} \pi^{10}}\left(\operatorname{sech}\{(2 p+1) \pi\}+\tanh \{(2 p+1) \pi\} \tanh \left\{\frac{(2 p+1) \pi}{2}\right\}\right)\right\} \operatorname{Pr}
\end{aligned}
$$




$$
\approx-2.015 \times 10^{-7} \mathrm{Pr}
$$

while as $\operatorname{Pr} \rightarrow \infty$,

$$
f(\operatorname{Pr}) \sim-\frac{131}{2^{9} \times 3^{5} \times 5^{2} \times 7 \times 11} \operatorname{Pr}^{2}
$$

\section{References}

Grundy, R.E. \& Kay, A. The asymptotics of blow-up in inviscid Boussinesq flow and related systems. IMA J. Appl. Math. 68, $47-81$ (2003).

Grundy, R.E. \& McLaughlin, R. 1997 Global blow-up of separable solutions of the vorticity equation. IMA J. Appl. Math. 59, 287-307.

Jacqmin, D. 1991 Frontogenesis driven by horizontally quadratic distributions of density. $J$. Fluid Mech. 228, 1-24.

Kay, A. 1992 Frontogenesis in gravity-driven flows with non-uniform density gradients. J. Fluid Mech. 235, 529-556.

Kay, A. 2004 Inertial oscillations and frontogenesis driven by a quadratic horizontal density variation. Geophys. Astrophys. Fluid Dyn. 98, 297-323.

Ohkitani, K. \& Gibbon, J.D. 2000 Numerical study of singularity formation in a class of Euler and Navier-Stokes flows. Phys. Fluids 12, 3181-3194.

Oosthuizen, P.H. and Paul, J.T. 1996 A numerical study of the steady state freezing of water in an open rectangular cavity. Int. J. Num. Meth. Heat Fluid Flow 6, 3-16. 